\title{
An Improved BP Neural Network Algorithm for Evaluating Food Traceability System Performance
}

\author{
Weiya Guo \\ School of Economics and Management, Beijing Jiaotong University, Beijing 100044, China
}

407706483@qq.com

\begin{abstract}
Keywords: BP neural network algorithm, Performance evaluation, food traceability system,
\end{abstract} trigonometric function.

\begin{abstract}
BP neural network algorithm has powerful calculation ability, but the algorithm has some shortages such as low convergence which limits its application, so improving BP algorithm has become a matter of concern in the fields related. Based on analyzing improvement methods wildly used today, the paper presents a new BP neural network algorithm and applies it to evaluate food traceability system performance. Firstly, the paper improves the BP algorithm through changing learning rate, trigonometric function to simplify the original calculation structure; secondly, the calculation step of the improved BP algorithm is redesigned to speed up its convergence. Finally, the paper conducts the theoretical analysis of the calculation performance of the improved algorithm and applies it to evaluate food traceability system performance, the theoretical analysis and experimental evaluation results show that the improved algorithm can improve evaluation accuracy and algorithm calculation efficiency and can be used for evaluating food traceability system performance practically.
\end{abstract}

\section{Introduction}

Artificial neural network is a nonlinear system which simulates cerebrum information processing algorithm. It has powerful distributed information storage, parallel processing and adaptive learning ability. BP network contains the most essence part of neural network theory. Owing to simple structure and technical mature, it has been widely applied in pattern recognition, intelligent control and other areas. However, BP algorithm uses the steepest descent algorithm, thus there are two main shortcomings of slow convergence rate and easy to fall into local minimum [1].

Studies on the improvement theory of BP neural network are comprised of the following five aspects. (1) Improve the gradient of excitation function, standard BP algorithm adopts sigmoid non-linear function as characteristic function of neuron, so if the net input of neuron becomes too large or too small, the output will enter saturation region, the error at this time may be very large. Error curved surface will show non-convergence. Therefore, rate of convergence of the model can be accelerated through improving excitation function [2]; (2) Improve error curved surface; Standard BP algorithm adopts square-error as objective function and uses gradient descent to minimize the total error function. With the increase of learning times, function slows in approximation velocity, failing to guarantee the approximation accuracy of height non-linear sample. If only considering that error term may have over-fitting phenomenon, causing poor generalization ability of network, many scholars have put forward improvement methods[3]; (3) Selection of initial weight value of network; Under normal conditions, the selection of initial weight value of BP algorithm is a set of random numbers among $[0,1]$, through repeated adjustment, obtaining stable weight value. But such kind of selection method may cause the network to fall into local minimum, resulting in failing to get optimal solution. Therefore, many scholars optimize the initial value through composite algorithm, also achieving favorable effects [4]; (4)Improving and optimizing algorithm; Standard BP algorithm adopts gradient descent to adjust weight value, making the training easy to fall into local minimum and slow in rate of convergence; such kind of method to improve and optimize algorithm is widely 
used at home and abroad, basically carrying out the improvement through the optimization theory of applied mathematics [5].

The paper improves BP algorithm through changing learning rate, trigonometric function, redesigning calculation step to speed up the calculation and simplify the structure of the original BP neural network algorithm. In so doing, not only the problem of convergence speed of BP algorithm has been solved, but also the simplicity of the algorithm structure are ensured.

\section{Redesigning BP Neural Network Algorithm}

\subsection{Working Principles BP Neural Network Algorithm.}

The learning process of BP neural network is a kind of guided learning method. First, the tutor shall set a desired output for each input mode, then input actual learning memory mode on network, propagating from input layer to output layer through intermediate layer. The difference between actual output and desired output is error. According to the criterion of minimum mean square error, the process that output layer amends link weight value layer by layer from the intermediate layer is called "back propagation of error". With the alternate repetition of "forward propagation of mode" and "back propagation of error", the actual output of network gradually approximates towards respectively corresponding desired output, and the accuracy of network's response to input mode is increasing. BP neural network, through such learning process, determines the link weight values among all the layers to start the work [6].

\subsection{Improvement with Change Learning Rate.}

Generally, when neural network model enters later phase of training, the link weight values among neurons become more stable; at this time, the learning rate shall be smaller, as larger learning rate is easy to make the modification process of weight value $W$ oscillate. While at the beginning of training, in order to accelerate the rate of convergence of network, the learning rate is often adjusted to a large value, which makes it necessary to dynamically change learning rate in learning process. The criterion for changing learning rate is to check whether the modification value of weight value actually reduces the error function. If yes, it means that the adopted learning rate is too small, and another increment can be added; if no, the value of learning rate shall be reduced. Optimization adjustment formula for changing learning rate method sees formula 1 , in which $\eta$ is learning rate, $l$ is training times, $E$ is error function, $\alpha, \beta$ and $k$ are rate factors, the values of which in this thesis are $\alpha=1.02, \beta=0.8, k=1.04$ [4].

$$
\eta_{l+1}=\left\{\begin{array}{c}
\alpha \eta_{l}, \alpha \geq 1, E_{l+1} \prec E_{l} \\
\beta \eta_{l}, \beta \leq 1, E_{l+1} \succ E_{l} \\
\eta_{l}, \quad \text { other }
\end{array}\right.
$$

In order to avoid that model training falls into slight local minimum point, momentum can be considered to be introduced; weighting adjustment formula with momentum is formula 2.

$$
E=\frac{1}{2} \sum_{q=1}^{Q} E_{q}=\frac{1}{2} \sum_{q=1}^{Q} \sum_{k=1}^{l}\left(d_{q, k}-v_{q, k}\right)^{2}
$$

And start the back propagation of error after finishing the learning of all the training samples, i.e. updating link weight values of each layer. WhenBP neural network algorithm is applied in batch adoption, its quantity of samples shall be more than 100 .

\subsection{Improvement with Trigonometric Function Design.}

This paper proposes to adopt trigonometric function to substitute $s$ type function, as shown in formula 3 .

$$
f(x)=0.5 \sin (\lambda x)+0.5
$$

In which, according to the experience, the value of $\lambda$ is among $[1.2,1.8]$; and the simulation result in this thesis shows that adopting the trigonometric function to be the excitation function of BP neural network achieves remarkable results on the global optimization, but not that obvious in the 
improvement of learning rate of network. Through further analysis and test, it is found that the reason why learning rate is not obviously enhanced is that there is no connection between two parameters in the above formula: 0.5 and $\lambda$; thus if change formula 3 into formula $4[6]$.

$$
f(x)=(0.5 / \lambda) \sin (\lambda x)+0.5 / \lambda
$$

In this way, while the period of function $f(x)$ is changed, the amplitude can be changed with the change of function period, thus changing the excitement degree of two connected neurons in layers, i.e. changing corresponding link weight values, in which the value of $\lambda$ is still among[1.2,1.8]; as a result, it can both guarantee global optimization of network and obviously enhance learning rate.

\subsection{Improvement with Calculation Step.}

The training step of improved BP neural network algorithm can be listed as follows. (1) Initial Population, including the population size and the initialization of each weight (generate according to the method for neural network to generate initial weight), and encode it;(2) Calculate the selection probability of each individual and sort them; (3) Select good individual to enter next generation population according to spinning roulette wheel selection strategy; (4) In the new generation population, select adaptive individual to carry out crossover and mutation operation according to adaptive crossover probability and mutation probability to generate new individual; (5) Insert the new individual into the population and calculate the fitness of new individual; (6) Immigration operator operation. Judge whether there is "prematurity phenomenon", if there is, immigration strategy shall be adopted and turn to step (2);(7) If the satisfactory individual is found, it shall be ended; otherwise, turn to (2).After reaching required performance indicator, decode the optimal individual in final population, then the optimized network connection weight can be obtained.

\section{Performance Analysis of the Improved BP Algorithm}

This paper applies BP algorithm to the approximation problem of non-linear function to prove the effectiveness of the improved algorithm. Approximate the non-linear function expression $\mathrm{y}=\left(\sin x_{1}+e^{x_{2}}\right) / 3$ with BP neural network, among which $x_{1} \in(0,20), x_{2} \in(0,2)$, adopting the input of sample set as $x_{1}=0: 0.5: 20, x_{2}=0: 0.05: 2$, desired output as $d=y\left(x_{1}, x_{2}\right)$, the 21 ones in which as training samples, and the rest 20 ones as test samples. Figure 1 shows the iterations performance of BP neural network algorithm before and after improvement in which figure 1 (a) means iterations of original BP algorithm and figure 1 (b) means iterations of improved BP algorithm.

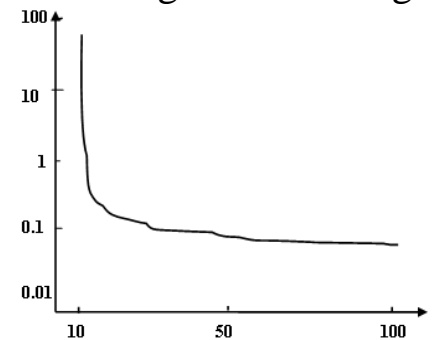

(a)

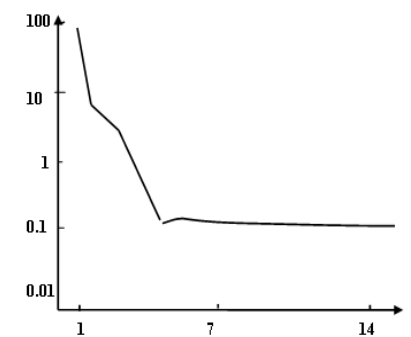

(b)

Fig. 1 The iterations comparison of BP algorithm before and after improvement.

\section{Algorithm Application in Evaluating Food Traceability System}

\subsection{Evaluation Indicator System Design.}

The paper takes pork traceability system for example and applies the improved BP algorithm to evaluate the performance of food traceability system. Food (pork) traceability system evaluation is a complicated and comprehensive operation system constituted by multiple elements, the numerous elements and subsystems of which exist in different forms, jointly assembly and forming competitiveness. This paper, based on analyzing attributes and functions of pork traceability system, uses BSC (Balanced Score Card, BSC) to analyze the concrete requirements of the performance 
evaluation of traceability system of pork supply chain, and builds a new performance evaluation indicator system from four dimensions of financial investment and output, system construction and management, customer service, learning and development. The designed performance evaluation indicator system is an extensive and scientific system and includes four hierarchies, four categories, nine second-class indicators, twenty-six third-class indicators. Limited the space of the paper, the specific contents of the indicators are omitted here.

\subsection{Experiment Results and Analysis.}

With C language, the presented BP algorithm is realized. The pork traceability system is taken as experimental data to build experimental database to evaluate food traceability system performance. The pork traceability systems of supply chain are named A, B and C respectively.

Table 1 and Table 2 show the specific evaluation results. Table 1 shows part of the intermediate evaluation results of the presented algorithm in the paper, and table 2 shows the evaluation results of ordinary multi-layer fuzzy comprehensive evaluation model [7], ordinary BP neural network algorithm [3] and the method presented in the paper. The former two algorithms are wildly used in complex system evaluation for its relatively high evaluation accuracy. The experiment is conducted through PC. PC configurations are as follows: P4 2.5G CPU and 512M memories.

Limited to paper space, the evaluation of intermediate results is omitted here, table 2 only provides parts of evaluation results and final comprehensive evaluation results.

Table 1 Part evaluation results of different pork traceability systems

\begin{tabular}{cccccc}
\hline & $\begin{array}{c}\text { Financial investment } \\
\text { and output }\end{array}$ & $\begin{array}{c}\text { System } \\
\text { construction and } \\
\text { management }\end{array}$ & $\begin{array}{c}\text { Customer } \\
\text { service }\end{array}$ & $\begin{array}{c}\text { Learning and } \\
\text { development }\end{array}$ & $\begin{array}{c}\text { Final } \\
\text { evaluation }\end{array}$ \\
\hline A & 3.236 & 3.357 & 3.902 & 2.408 & 3.214 \\
B & 3.779 & 3.625 & 4.132 & 3.939 & 3.967 \\
C & 4.137 & 4.421 & 4.607 & 4.106 & 4.398 \\
\hline
\end{tabular}

Table 2 Evaluation performance of different algorithms

\begin{tabular}{|c|c|c|c|}
\hline Algorithm & $\begin{array}{l}\text { Method in the } \\
\text { paper }\end{array}$ & $\begin{array}{l}\text { Ordinary BP evaluation } \\
\text { method }\end{array}$ & $\begin{array}{l}\text { Ordinary fuzzy } \\
\text { evaluation method }\end{array}$ \\
\hline Accuracy rate & $92.89 \%$ & $80.20 \%$ & $74.38 \%$ \\
\hline Time consuming (s) & 12 & 587 & 13 \\
\hline
\end{tabular}

\section{References}

[1] Chen Q., Huang K. M. A BP neural network realization in the measurement of material permittivity. Journal of Software. Vol. 33 (2011) No. 6, p. 1089-1095.

[2] Qian Q. L., Chen B. A color matching method based on machine learning. Chinese Journal of Instrument. Vol. 29 (2010) No. 9, p. 529-551.

[3] Kadhi C. J., Hadjar K. B. A mobile agent and artificial neural networks for intrusion detection. Journal of Computer. Vol. 41 (2014) No. 12, p. 1356-1363.

[4] Amandeal J. S., Shiun S. L. Research the improvement of BP neural network and its Application. Journal of Modern Mathmatics. Vol. 23 (2012) No. 7, p. 52-61.

[5] Souza M. T., Monterio Q. V. Research the methods of complex system evaluation. Journal of Management Science. Vol. 88 (2013) No.1, p. 23-37.

[6] Gao Z. Y. A study on roughness coefficient using BP neural network. Journal of Statistics. Vol. 72 (2014) No. 3, p. 66-79.

[7] Yueh Y. S. Fuzzy quality attributes for evaluating internet public opinion performance. Journal of Simulation Calcation. Vol. 15 (2013) No. 10, p. 931-939. 